\title{
Efeito agudo dos exercícios resistidos sobre o desempenho da amplitude articular
}

\author{
Acute effect of resistance exercises on joint range-of-motion \\ performance
}

\author{
Lennart da Silva Novaes Neto'; Claudio Melibeu Bentes²; Humberto Lameira Miranda33; Rodolfo de \\ Alkmim Moreira Nunes ${ }^{4}$ Thiago Matassoli Gomes5; Jefferson da Silva Novaes ${ }^{6}$ \\ ${ }^{1}$ Mestre em Educação Física - Universidade Federal do Rio de Janeiro - EEFD/UFRJ, Pesquisador do projeto PET - saúdeVS - UFRJ \\ Rio de Janeiro, RJ - Brasil. \\ ${ }^{2}$ Mestre em Educação Física - Universidade Federal do Rio de Janeiro - EEFD/UFRJ, Professor Substituto da UFRJ - Escola de \\ Educação Física e Desportos - Depto de Ginástica, Pesquisador do projeto PET - saúdeVS - UFRJ. Rio de Janeiro, RJ - Brasil. \\ 'Doutor em Engenharia Biomédica - Universidade do Vale do Paraíba - UNIVAP, Professor Adjunto da UFRJ - Escola de Educação \\ Física e Desportos - Depto de Ginástica, Coordenador do projeto PET - saúdeVS - UFRJ, Coordenador da Pós-Graduação em \\ Musculação e Treinamento de Força - UFRJ. Rio de Janeiro, RJ - Brasil. \\ ${ }^{4}$ Doutor em Ciências da Saúde - Universidade Federal do Rio Grande do Norte - UFRN, Professor Adjunto - Universidade do Estado \\ do Rio de Janeiro - UERJ. Rio de Janeiro, RJ - Brasil. \\ Mestre em Educação Física - Universidade Federal do Rio de Janeiro - EEFD/UFRJ, Professor da Pós-Graduação Lato Sensu do Centro \\ Universitário de Volta Redonda - UNIFOA, Pesquisador do projeto PET - saúdeVS - UFRJ. Rio de Janeiro, RJ - Brasil. \\ ${ }^{6}$ Doutor em Educação Física - Universidade Gama Filho - UGF, Professor Adjunto da UFRJ - Escola de Educação Física e Desportos - \\ Depto de Ginástica. Rio de Janeiro, RJ - Brasil.
}

Endereço para correspondência Thiago Matassoli Gomes

Av. Brigadeiro Trompowsky, s/n - Cidade Universitária Ilha do Fundão 21941-590 - Rio de Janeiro - RJ [Brasil] thiagogom@gmail.com

\begin{abstract}
Resumo
Objetivo: Comparar o efeito de diferentes intensidades de exercícios resistidos sobre o desempenho da amplitude articular de jovens treinados. Métodos: Após a realização dos testes iniciais (força e flexibilidade), os voluntários foram divididos aleatoriamente nas seguintes condições experimentais: (a) protocolo de força com $100 \% 1 \mathrm{RM}$ + teste de flexibilidade (G100); (b) protocolo de força com $80 \%$ $1 \mathrm{RM}+$ teste de flexibilidade (G80); (c) protocolo de força com $60 \% 1 \mathrm{RM}+$ teste de flexibilidade (G60) e (d) protocolo de força com 40\% 1RM + teste de flexibilidade (G40). Para determinar os efeitos das quatro condições experimentais sobre a variável dependente (amplitude articular), foi realizada a análise de variância de um fator (one-way ANOVA). Para determinar as diferenças específicas foi realizado o teste post hoc de Bonferoni. Resultados: Dentre todas as análises, a condição G80 foi a que promoveu maiores alterações significativas sobre o desempenho da amplitude articular. Conclusão: Diferentes intensidades de exercícios resistidos provocam aumentos significativos sobre a resposta da amplitude articular em indivíduos jovens treinados.
\end{abstract}

Descritores: Amplitude de movimento articular; Flexibilidade; Força muscular; Músculo esquelético.

\begin{abstract}
Objective: To compare the effect of different intensities of resistance exercises on joint range-of-motion performance of young male trainees. Methods: After completion of the initial tests (strength and flexibility), the volunteers were randomly assigned into the following experimental conditions: (a) protocol with $100 \% 1 \mathrm{RM}$ strength + flexibility test (G100), (b) protocol with 80\% 1RM strength + flexibility test (G80), (c) protocol strength of $60 \% 1 \mathrm{RM}+$ flexibility test (G60), and (d) protocol with $40 \%$ 1RM strength + flexibility test (G40). To determine the effects of the four experimental conditions on the dependent variable (joint range-of-motion), a one-way ANOVA was performed. To determine the specific differences the Bonferoni post hoc test was performed. Results: Among all analyzes, the condition G80 was promoted major significant changes on the joint range-of-motion performance. Conclusion: Different intensities of resistance exercise cause significant increases on the joint range-of-motion amplitude performance in young male trainees.
\end{abstract}

Key words: Flexibility; Muscle Strength; Range of motion; Skeletal muscle. 


\section{Introdução}

Níveis adequados de força muscular e flexibilidade são fundamentais para o bom funcionamento musculoesquelético, contribuindo para a preservação de músculos e articulações saudáveis ao longo da vida ${ }^{1}$. Contudo, o declínio da força muscular e o dos níveis de flexibilidade vão gradativamente dificultando a realização de diferentes tarefas cotidianas, levando, muitas vezes, à perda precoce de autonomia funcional ${ }^{2}$.

A prática regular de programas de exercícios físicos voltados para o desenvolvimento ou manutenção da força muscular e da flexibilidade ou, até mesmo, de outros importantes componentes da aptidão física relacionados à saúde pode exercer papel extremamente relevante ao longo da vida ${ }^{3,4,5}$. Entretanto, a exata demanda para a prescrição dessas duas valências em um programa de atividade física supervisionado não aparece de maneira clara na literatura.

A realização de exercícios resistidos (ER) potencializa, inquestionavelmente, a força, a potência e a hipertrofia muscular nos mais diferentes tipos de indivíduos ${ }^{1,3,4,5}$. Além disso, a realização continuada de um treinamento de força parece provocar aumentos significativos no desempenho da flexibilidade em diferentes articulações. Barbosa et al. ${ }^{6}$ observaram aumentos significativos de $13,9 \%$ no desempenho do teste de sentar e alcançar de idosas inativas após a realização de dez semanas de ER. Fatouros et al. ${ }^{7}$ investigaram o efeito de 24 semanas de ER realizado em diferentes intensidades sobre o desempenho da amplitude articular em idosos inativos. Os autores observaram uma demanda de aumentos entre $3 \%$ e $28 \%$ no desempenho da flexibilidade em diferentes articulações. As maiores diferenças ocorreram no grupo que realizou os ERs com maior intensidade. Além disso, ao analisar o destreinamento, os autores observaram que o grupo que treinou com maior intensidade obteve menores perdas na amplitude articular. Assim, observa-se que pode existir uma relação de dependência entre o desempenho da flexibilidade e a intensidade dos $\mathrm{ER}^{7}$.

Estudando jovens adultos, Monteiro et al. ${ }^{8}$ investigaram os efeitos dos ER sobre o desempenho da flexibilidade em mulheres sedentárias de meia idade (35 a 39 anos). Os movimentos de adução horizontal do ombro, flexão e extensão do tronco e do quadril apresentaram aumentos significativos (37,3\%; 146,2\%; $135,6 \%$; $15,4 \%$ ) após a realização de dez semanas de ER. Santos et al. ${ }^{9}$ observaram que os métodos alternado por segmento e agonista-antagonista promoveram aumentos significantes $(p<0,05)$ na amplitude articular de mulheres sedentárias. Ao analisar os efeitos dos ER, sendo realizados de maneira isolada ou combinada com exercícios de alongamento, Simão et al. ${ }^{10}$ observaram aumentos no desempenho da flexibilidade em todas as comparações efetuadas. Os autores concluíram que somente a realização de ER promovem aumentos significativos na flexibilidade. Entretanto, Nóbrega et al. ${ }^{11}$ não verificaram diferenças significantes na amplitude articular de jovens inativos, após 12 semanas de ER realizado de maneira isolada. Os autores demonstraram aumentos importantes na amplitude articular para o treinamento específico de flexibilidade $(+33 \%$; $p<0,001)$ ou em combinação do treino de flexibilidade com o de força $(+18 \%$; $\mathrm{p}<0,001)$.

Há muitos fatores que podem influenciar a flexibilidade, como o aumento da amplitude de movimento, incluindo o grau de condição física, idade, especificidade do treinamento e variáveis de prescrição metodológica (ordem e número de exercícios, séries, repetições, intervalos de descanso e sistema de treinamento) $)^{8,11,12}$. Embora a realização continuada dos ER melhore o desempenho da flexibilidade em diferentes articulações, permanecem dúvidas sobre o impacto desses exercícios no desempenho da flexibilidade em jovens treinados. Em vista disso, o objetivo neste estudo foi comparar o efeito agudo de diferentes intensidades de ER (40\%, 60\%, 80\% e $100 \%$ de 1 RM) sobre o desempenho da amplitude articular de jovens treinados. 


\section{Material e métodos}

\section{Sujeitos}

Foram selecionados 15 indivíduos do sexo masculino $(22,9 \pm 2,18$ anos; $4 \pm 6,78 \mathrm{~kg} ; 180,8$ $\pm 2,05 \mathrm{~cm})$, voluntários, aparentemente saudáveis, com frequência semanal de treinamento de no mínimo três vezes por semana e experiência mínima de um ano na realização de ER. Todos os participantes do estudo assinaram o Termo de Consentimento Livre e Esclarecido, que continha informações pertinentes ao experimento e assegurava a sua privacidade. $\mathrm{O}$ estudo registrado, sob o número 101/2011, foi aprovado pelo Comitê de Ética em Pesquisa da Universidade Federal do Rio de Janeiro (UFRJ). Todos os princípios de ética foram respeitados de acordo com a Resolução 196/96, do Conselho Nacional de Saúde.

\section{Desenho experimental}

Cada voluntário realizou seis visitas (Figura 1) não consecutivas com intervalo de 48 horas entre elas. Todos os participantes efetuaram os testes no mesmo período do dia durante todo o procedimento experimental. Na primeira visita, foram feitas as mensurações da composição corporal (estatura e massa corporal), aplicado o questionário PAR-Q, e realizados os testes de flexibilidade e de 1RM para os exercícios supino horizontal (SH) e leg press (LP). $\mathrm{Na}$ segunda visita, efetuaram-se os retestes de flexibilidade e de 1RM. Da terceira a sexta visitas, os voluntários foram divididos aleatoriamente com entrada contrabalançada, nas seguintes condições experimentais: (a) protocolo de força com $100 \%$ carga de 1RM + teste de amplitude articular (G100); (b) protocolo de força com $80 \%$ da carga de 1RM + teste de amplitude articular (G80); (c) protocolo de força com $60 \%$ da carga de 1RM + teste de amplitude articular (G60) e (d) protocolo de força com $40 \%$ da carga de $1 \mathrm{RM}+$ teste de amplitude articular (G40). Todos foram instruídos a não executar qualquer tipo de exercício de força e de flexibilidade para o grupamento muscular envolvido nos testes, 48 horas antes do início destes. Para todas as intensidades, os voluntários foram orientados a realizar o maior número de repetições para cada um dos exercícios (SH e LP) até a falha concêntrica. O intervalo observado para o desempenho dos testes de força entre os exercícios não foi inferior a três minutos.

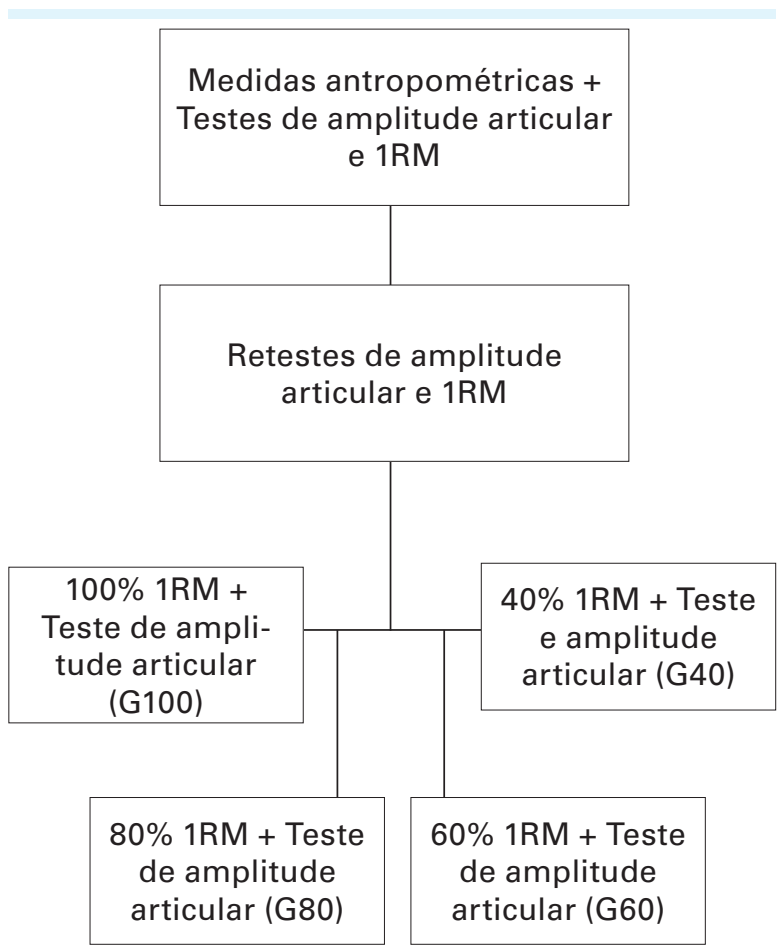

Figura 1: Desenho experimental

\section{Teste de amplitude articular}

Foi realizado um teste e um reteste de flexibilidade para verificação da reprodutibilidade dos resultados. A flexibilidade foi medida também logo após a execução dos protocolos experimentais (G40, G60, G80, G100). Foram realizadas as medidas angulares por meio de goniometria, segundo o protocolo adaptado de Norkin e White ${ }^{13}$, com amplitude máxima em seis movimentos articulares, sendo: (a) flexão do ombro; (b) extensão do ombro; (c) abdução horizontal do ombro; (d) adução horizontal do ombro; (e) flexão do quadril e (f) extensão do quadril. Todas as medidas foram coletadas do lado direito. 
Para medir a flexibilidade, o avaliador conduziu o segmento do avaliado até a limitação mecânica do movimento. As medidas foram efetuadas sempre na mesma hora do dia. Para os testes de flexibilidade, foi utilizado um goniômetro da marca Lafayette (modelo Sammons Preston Rolyan \#7514).

\section{Teste de IRM}

Como forma de aquecimento, cada indivíduo realizou uma série de cinco a dez repetições com uma carga equivalente a $40 \%$ do peso máximo percebido. Após um minuto de repouso, foi realizada a segunda série entre três e cinco repetições com $60 \%$ a $80 \%$ do máximo percebido. Em seguida, deu-se início aos testes, em que até três tentativas puderam ser realizadas, sendo o peso ajustado sempre antes de cada tentativa. O tempo de recuperação entre as tentativas foi padronizado em cinco minutos. Quando o avaliado não conseguia mais realizar o movimento de forma correta o teste era interrompido, sendo registrada como carga máxima, aquela obtida na última execução completa. Algumas estratégias foram adotadas no sentido de reduzir a margem de erro da coleta, foram elas: (a) instruções padronizadas foram oferecidas antes dos testes de modo que o avaliado tivesse ciência de toda a rotina que envolvia a coleta de dados; (b) o participante foi instruído sobre a técnica para realizar o exercício; (c) o avaliador estava atento quanto à posição adotada pelo praticante no momento da execução dos movimentos; (d) todos os sujeitos receberam encorajamentos verbais durante as tentativas; (e) os testes foram realizados na mesma hora do dia em todas as sessões. O tempo de intervalo entre os exercícios foi o de no mínimo 30 minutos.

\section{Tratamento estatístico}

O teste de Kolmogorov-Smirnov foi utilizado para testar a gaussianidade das variáveis de caracterização da amostra. $\mathrm{O}$ cálculo dos incrementos percentuais nos valores da amplitude articular após as condições experimentais foi realizado por meio do tamanho do efeito (diferença entre a média pré e a pós-teste dividida pelo desvio-padrão pré-teste ), proposto por Rhea ${ }^{14}$. Para determinar os efeitos das quatro condições experimentais sobre a variável dependente (amplitude articular), foi realizada uma análise de variância de um fator (one-way ANOVA). Para determinar as diferenças específicas foi realizado o teste post hoc de Bonferoni. As análises estatísticas foram realizadas a partir do pacote de programas estatísticos SPSS 14.0 (SPSS Inc., EUA). Para todas as análises foi adotado um nível crítico de significância de $p<0,05$.

\section{Resultados}

Os movimentos de flexão, abdução e adução horizontal de ombro apresentaram aumentos significativos $(\mathrm{p}<0,05)$ para a condição experimental G80, quando comparada com o momento pré-teste (Tabela 1). Ainda foram observadas diferenças significantes $(p<0,05)$ para o movimento de abdução horizontal, entre a condição G80 $(155,33 \pm 13,55)$ e a G40 $(140,67 \pm$ 9,97); e na adução horizontal, quando comparadas as condições experimentais G60 $(78 \pm 4,55)$ com o momento pré-teste $(72,67 \pm 4,17)$, e G100 $(74,67 \pm 5,81)$ com G80 $(80,33 \pm 4,80)$ (Tabela 1). O movimento de extensão de quadril apresentou aumentos significativos $(\mathrm{p}<0,05)$ para a condição G80 $(69,67 \pm 8,95)$ em relação às condições pré-teste $(50 \pm 9,25)$, G60 (57 \pm 11,61) e G40 (56 \pm $8,90)$ (Tabela 1). O tamanho do efeito (Tabela 2) demonstra as diferenças na medida da flexibilidade após cada condição experimental.

\section{Discussão}

Neste estudo, investigaram-se os efeitos de diferentes intensidades de ER sobre a resposta da amplitude articular em indivíduos jovens treinados. De todas as condições experimentais analisadas, a condição G80 foi a que promoveu 
Tabela l: Média, desvio-padrão e $\Delta \%$ para todas as condições experimentais

\begin{tabular}{|c|c|c|c|c|c|c|c|c|c|}
\hline Movimentos & PRÉ & G100 & $\Delta \%$ & G80 & $\Delta \%$ & G60 & $\Delta \%$ & G40 & $\Delta \%$ \\
\hline $\begin{array}{l}\text { Flexão de ombro } \\
\text { (em graus) }\end{array}$ & $\begin{array}{c}174 \\
\pm 15,49\end{array}$ & $\begin{array}{l}183,67 \\
\pm 18,75\end{array}$ & 5,55 & $\begin{array}{c}194 \\
\pm 13,52^{*}\end{array}$ & 11,49 & $\begin{array}{c}184 \\
\pm 14,66\end{array}$ & 5,74 & $\begin{array}{l}180,33 \\
\pm 15,40\end{array}$ & 3,63 \\
\hline $\begin{array}{l}\text { Extensão de ombro } \\
\text { (em graus) }\end{array}$ & $\begin{array}{l}47,33 \\
\pm 7,98\end{array}$ & $\begin{array}{c}57,33 \\
\pm 12,08\end{array}$ & 21,12 & $\begin{array}{l}68,33 \\
\pm 9,19\end{array}$ & 44,36 & $\begin{array}{l}56,67 \\
\pm 9,94\end{array}$ & 19,71 & $\begin{array}{l}54,67 \\
\pm 7,66\end{array}$ & 15,49 \\
\hline $\begin{array}{l}\text { Adução horizontal de ombro } \\
\text { (em graus) }\end{array}$ & $\begin{array}{l}72,67 \\
\pm 4,17\end{array}$ & $\begin{array}{l}74,67 \\
\pm 5,81\end{array}$ & 2,75 & $\begin{array}{c}80,33 \\
\pm 4,80^{*} \neq\end{array}$ & 10,55 & $\begin{array}{c}78 \\
\pm 4,55^{*}\end{array}$ & 7,33 & $\begin{array}{c}77 \\
\pm 4,14\end{array}$ & 5,96 \\
\hline $\begin{array}{c}\text { Abdução horizontal de ombro } \\
\text { (em graus) }\end{array}$ & $\begin{array}{l}135,33 \\
\pm 9,90\end{array}$ & $\begin{array}{r}145,33 \\
\pm 15,86\end{array}$ & 7,38 & $\begin{array}{l}155,33 \\
\pm 13,55^{*}\end{array}$ & 14,77 & $\begin{array}{c}144 \\
\pm 9,85\end{array}$ & 6,40 & $\begin{array}{l}140,67 \\
\pm 9,97^{\dagger}\end{array}$ & 3,94 \\
\hline $\begin{array}{l}\text { Flexão de quadril } \\
\text { (em graus) }\end{array}$ & $\begin{array}{l}143,67 \\
\pm 8,54\end{array}$ & $\begin{array}{l}153,33 \\
\pm 10,96\end{array}$ & 6,72 & $\begin{array}{l}162,33 \\
\pm 9,03\end{array}$ & 12,99 & $\begin{array}{c}153 \\
\pm 8,61\end{array}$ & 6,49 & $\begin{array}{l}150,67 \\
\pm 8,42\end{array}$ & 4,87 \\
\hline $\begin{array}{l}\text { Extensão de quadril } \\
\text { (em graus) }\end{array}$ & $\begin{array}{c}50 \\
\pm 9,25\end{array}$ & $\begin{array}{c}60 \\
\pm 16,36\end{array}$ & 20 & $\begin{array}{l}69,67 \\
\pm 8,95^{*}\end{array}$ & 39,33 & $\begin{array}{c}57 \\
\pm 11,61^{\dagger}\end{array}$ & 14 & $\begin{array}{c}56 \\
\pm 8,90^{+}\end{array}$ & 12 \\
\hline
\end{tabular}

*Diferença significativa $(p<0,05)$ em relação ao momento PRÉ

tDiferença significativa $(p<0,05)$ em relação a G80

‡Diferença significativa $(p<0,05)$ em relação a $\mathrm{G} 100$

Tabela 2: Tamanho do efeito e magnitudes do desempenho da amplitude articular entre todas as condições experimentais

\begin{tabular}{ccccc}
\hline Movimentos & G100 vs Pré & G80 vs Pré & G60 vs Pré & G40 vs Pré \\
\hline Flexão de ombro & 0,6 & 1,2 & 0,6 & 0,4 \\
Magnitude & Pequeno & Moderado & Pequeno & Pequeno \\
Extensão de ombro & 1,2 & 2,6 & 1,1 & 0,9 \\
Magnitude & Moderado & Grande & Moderado & Moderado \\
Abdução horizontal de ombro & 1,0 & 2,0 & 0,8 & 0,5 \\
Magnitude & Moderado & Grande & Moderado & Pequeno \\
Adução horizontal de ombro & 0,4 & 1,8 & 1,2 & 1,0 \\
Magnitude & Pequeno & Grande & Moderado & Moderado \\
Flexão de quadril & 1,1 & 2,1 & 1,0 & 0,8 \\
Magnitude & Moderado & Grande & Moderado & Moderado \\
Extensão de quadril & 1,0 & 2,1 & 0,7 & 0,6 \\
Magnitude & Moderado & Grande & Moderado & Moderado \\
\hline
\end{tabular}

maiores alterações significativas sobre o desempenho da amplitude articular. É importante ressaltar que nenhuma das condições experimentais provocou diminuições significativas na amplitude articular de nenhuma das articulações envolvidas no artigo.

Nóbrega et al. ${ }^{11}$ não observaram aumentos significativos $(\mathrm{p}>0,05)$ na flexibilidade de jovens inativos, quando o treinamento de força foi realizado de maneira isolada. Os autores investigaram 43 jovens inativos durante 12 semanas, e os distribuíram em quatro grupos, a saber: (TFor) apenas treinamento de força; (TFle) somente treino de flexibilidade; (TFF) treinamento de força e flexibilidade e (GC) grupo controle. O método Flexitest $\mathrm{t}^{15}$ foi utilizado para avaliar a flexibilidade dos indivíduos. Os grupos treinaram duas vezes por semana. A flexibilidade aumentou de maneira significativa, quando treinada de maneira isolada $(+33 \%$; $\mathrm{p}<0,001)$ ou em combinação com o treinamento de força $(+18 \%$; $p<0,001)$. Em sua conclusão, os autores afirmam que o treino de força melhora a amplitude articular apenas quando combinado com um treinamento da flexibilidade, sendo improváveis alterações significativas ao ser efetuado de maneira isolada. Os resultados do atual estudo não corroboram os encontrados por 
Nóbrega et al. ${ }^{11}$. Importantes diferenças entre os trabalhos devem ser levadas em consideração ao realizar a comparação dos achados, como o fato de o método de avaliação da flexibilidade utilizado por Nóbrega et al. ${ }^{11}$ - apesar de validado e bastante difundido na literatura - ser extremamente subjetivo e dependente da acurácia e experiência do avaliador. Outra observação relevante tange a quantidade de vezes por semana (apenas duas) em que os indivíduos realizaram as sessões de treinamento. Estudos em que foram efetuadas pelo menos três sessões de treinamento semanais demonstraram melhoras na flexibilidade, quando os ER eram executados de maneira isolada ${ }^{8,9,10}$.

Os dados deste estudo corroboram os achados previamente descritos na literatura ${ }^{6-10}$, demonstrando que a realização de ER em intensidades próximas a $80 \% 1 \mathrm{RM}$ potencializa a flexibilidade em relação a outras intensidades. O tempo de tensão aplicado em cada série de ER parece proporcionar mudanças fisiológicas importantes, como a remodelação das moléculas de colágeno e elastina ${ }^{16}$. A amplitude do movimento seria influenciada pelo aumento do comprimento de um tecido, proporcionalmente à tensão aplicada. Tais aumentos de amplitude podem ser, ainda, decorrentes da melhoria das atividades neurais ${ }^{17,18}$. Uma das hipóteses relaciona-se às modificações nos mecanismos neuromusculares mediante estímulos nas unidades motora e consequente excitação dos motoneurônios alfa e gama ${ }^{19}$. Os efeitos neurais proporcionam, inicialmente, um aumento da amplitude de movimento antes do acionamento do sistema reflexo, com a posterior participação dos fusos e das ações inibitórias autogênica e recíproca, provocadas por contrações isométricas ocorridas durante todo o processo de aumento de comprimento e geração de tensão durante o alongamento ${ }^{17,18}$. Entretanto, esses efeitos, inicialmente importantes, parecem não produzir resultados significativos, quando comparados com a hipótese de redução das resistências mecânicas. Essa redução parece acontecer em virtude das mudanças nas pro- priedades viscoelásticas dos tecidos moles e conjuntivos, submetidos a estresses constantes durante o treinamento da flexibilidade ${ }^{20}$.

Fatouros et al. ${ }^{7}$ investigaram o efeito da realização de 24 semanas de ER sobre as respostas da flexibilidade em diferentes articulações de 58 idosos destreinados, os quais foram divididos aleatoriamente em quatro grupos, sendo: controle (GC, n=10); EF de baixa intensidade (BI, $40 \% 1 \mathrm{RM}, \mathrm{n}=14)$; ER de intensidade moderada (MI, 60\%1RM, n=12) e EF de alta intensidade (AI, $80 \% 1 \mathrm{RM}, \mathrm{n}=14)$. Os autores avaliaram a flexibilidade nas articulações do quadril, ombro, joelho e cotovelo pela técnica de goniometria; e a do tronco foi aferida mediante uma modificação do teste sentar e alcançar. A flexibilidade do tronco, cotovelo, ombro, joelho e quadril aumentou de maneira significativa nos grupos experimentais, sendo mais efetiva no MI (6-22\%) e no AI (8-28\%) do que no BI (3-12\%). Apesar de utilizar sujeitos com níveis de treinamento e idades diferentes (idosos destreinados vs jovens treinados), os resultados do estudo aqui apresentado confirmam os resultados de Fatouros et al. ${ }^{7}$, uma vez que o G80 apresentou aumentos na flexibilidade que variaram de $10,55 \%$ a $44,36 \%$, o que demonstra que a resposta do desempenho da flexibilidade, após a realização de ER, tem íntima relação com a intensidade do treinamento ${ }^{7}$.

Monteiro et al. ${ }^{8}$ verificaram o efeito dos ER na flexibilidade em 20 mulheres, entre 35 e 39 anos, inativas, durante dez semanas. As medidas da flexibilidade foram realizadas em dez movimentos articulares: flexão/extensão do ombro, adução/abdução horizontal do ombro, flexão do cotovelo, flexão/extensão do quadril, flexão do joelho, flexão/extensão do tronco. Dos quatro movimentos de ombro analisados, somente o movimento de adução horizontal apresentou um aumento significativo (37,3\%). Os movimentos de flexão e extensão das articulações do tronco e do quadril também apresentaram aumentos importantes (146,2\%; 135,6\%; 15,4\%; $23,6 \%$ respectivamente). Não foram verificadas diferenças significativas nas articulações do joelho e do cotovelo. Os autores sustentam a hipóte- 
se de que nas primeiras semanas de treinamento, a realização de exercícios de alongamento é necessária para a obtenção de ganhos na flexibilidade. Mesmo assim, obtiveram resultados significativos para quase todas as articulações analisadas, assim como observado neste estudo. Barbosa et al. ${ }^{6}$ também referem aumentos significativos $(13 \pm 9 \%)$ na flexibilidade de idosos, após a realização de dez semanas de ER. Apesar da utilização de diferentes métodos para a avaliação da flexibilidade, os dados desta pesquisa ratificam os achados de Barbosa et al. ${ }^{6}$, em que se observam aumentos na flexibilidade a partir de $10,55 \%$.

Santos et al. ${ }^{9}$ realizaram o primeiro estudo para analisar se o treinamento de resistência moderadamente intenso melhora a flexibilidade de mulheres sedentárias. As voluntárias foram divididas nos seguintes grupos: grupo agonista / antagonista (AA), alternado por segmento (AST) e grupo controle (GC). Os grupos AA e AST aumentaram significativamente a força e a flexibilidade do pré para o pós-teste, quando comparados ao GC $(p<0,05)$. O AST aumentou a força e a flexibilidade mais do que o AA (p < $0,05)$, em todas as medidas. Esse estudo mostra que o exercicio resistido pode melhorar a flexibilidade em mulheres jovens sedentárias em oito semanas.

Após 16 semanas de treinamento, Simão et al. ${ }^{10}$ examinaram os ganhos de força e flexibilidade em 80 mulheres sedentárias que foram separadas aleatoriamente em quatro grupos, sendo: força $(n=20)$; força e flexibilidade $(n=20)$; flexibilidade $(n=20)$ e grupo controle $(n=20)$. O teste utilizado para medir a flexibilidade foi o de sentar e alcançar, e o teste de 10RM foi utilizado nos exercícios leg press e supino horizontal para verificar a força. Os resultados mostram que os três grupos obtiveram melhorias significativas em comparação à linha de base e ao grupo controle, porém não foi encontrada diferença significante entre os grupos. Em conclusão, os autores demonstram que o ER contribui para a melhoria e manutenção da flexibilidade sem a inclusão de qualquer tipo de alongamento, mas
ER e alongamento podem ser prescritos juntos para obter otimização dos resultados.

Diante do exposto, os resultados apresentados demonstram uma significativa melhora da flexibilidade para diversos movimentos articulares, quando o treinamento de força é realizado a $80 \%$ da carga de $1 \mathrm{RM}$. Insta salientar, que nenhuma das demais intensidades analisadas provocou diminuição - de forma aguda - no desempenho da amplitude articular.

\section{Conclusão}

Os resultados deste estudo sugerem que diferentes intensidades de ER provocam aumentos significativos na resposta da amplitude articular em indivíduos jovens treinados. Entretanto, de todas as condições experimentais analisadas, a condição G80 foi a que promoveu maiores alterações significativas sobre o desempenho da amplitude articular. Recomenda-se que outras pesquisas, utilizando diferentes intensidades e movimentos articulares, sejam realizadas a fim de elucidar e reforçar as questões apresentadas pela literatura científica.

\section{Referências}

1. Novaes JS. Ciência do treinamento dos exercícios resistidos. São Paulo: Phorte; 2008.

2. Roberts MD, Kerksick CM, Dalbo VJ, Hassell SE, Tucker PS, Brown R. Molecular attributes of human skeletal muscle at rest and after unaccustomed exercise: an age comparison. J Strength Cond Res. 2010;24(5):1161-8.

3. American College of Sports Medicine. Progression models in resistance training for healthy adults. Med Sci Sports Exerc. 2002;34(2):364-80.

4. American College of Sports Medicine. Progression models in resistance training for healthy adults. Med Sci Sports Exerc. 2009;41(3):687-708. 
5. Garber CE, Blissmer B, Deschenes MR, Franklin BA, Lamonte MJ, Lee MD, et al. Quantity and quality of exercise for developing and maintaining cardiorespiratory, musculoskeletal, and neuromotor fitness in apparently healthy adults: guidance for prescribing exercise. Med Sci Sports Exerc. 2011;43(7):1334-59.

6. Barbosa AR, Santarém JM, Filho WJ, Marucci MFN. Effects of resistance training on the sit-andreach test in elderly women. J Strength Cond Res. 2002;16(1):14-8.

7. Fatouros IG, Kambas A, Katabrasas I, Leontsini D, Chatzinikolaou A, Jamurtas AZ, et al. Resistance training and a detraining effects on flexibility performance in the elderly are intensity-dependent. J Strength Cond Res. 2006;20(3):634-42.

8. Monteiro WD, Simão R, Polito MD, Santana CA, Chaves RB, Bezerra E, et al. Influence of strength training on adult women's flexibility. J Strength Cond Res. 2008;22(3):672-7.

9. Santos E, Rhea MR, Simão R, Dias I, Salles BF, Novaes J, et al. Influence of moderately intense strength training on flexibility in sedentary young women. J Strength Cond Res. 2010;24(11):3144-9.

10. Simão R, Lemos A, Salles B, Leite T, Oliveira E, Rhea $\mathrm{MR}$, et al. The influence of strength, flexibility, and simultaneous training on flexibility and strength gains. J Strength Cond Res. 2011;25(5):1333-8.

11. Nóbrega ACL, Paula KC, Carvalho AC. Interaction between resistance training and flexibility training in healthy young adults. J Strength Cond Res. 2005;19(4):842-6.
12. Lemos A, Simão R, Polito M, Salles B, Rhea MR, Alexander J. The acute influence of two intensities of aerobic exercise on strength training performance in elderly women. J Strength Cond Res. 2009;23(4):1252-7.

13. Norkin CC, White DJ. Medida do movimento articular: manual de goniometria. $2^{\mathrm{a}}$ ed. Porto Alegre: Artes Médicas; 1997.

14. Rhea MR. Determining the magnitude of treatment effects in strength training research through the use of the effect size. J Strength Cond Res. 2004;18(4):918-20.

15. Araújo CGS. Flexitest: an innovative flexibility assessment method. Champaign, IL: Human Kinetics; 2004.

16. Taylor DC, Dalton JD, Seaber AV, Garrett WE. Viscoelastic properties of muscle-tendon units. The biomechanical effects of stretching. Am J Sports Med. 1990;18(3):300-9.

17. Kubo K, Kanehisa H, Kawakami Y, Fukunaga T. Elasticity of tendon structures of lower limbs in sprinters. Acta Physiol Scand. 2000a;168(2):327-35.

18. Kubo K, Kanehisa H, Kawakami Y, Fukunaga T. Elastic properties of muscle-tendon complex in longdistance runners. Eur J Appl Physiol. 2000b;81(3):181-7.

19. Maior AS, Alves A. A contribuição dos fatores neurais em fases iniciais do treinamento de força muscular: uma revisão bibliográfica. Rio Claro: Motriz; 2003.

20. Burke DG, Culligan LE. The theoretical basis of proprioceptive neuromuscular facilitation. J Strength Cond Res. 2000;14(4):496-500. 